\title{
EL LEGADO DE LAS VOCES Y LOS ESCRITOS: SU VALOR COMO TESTIMONIO DE LA CULTURA ESCOLAR
}

\author{
Alejandro Mayordomo ${ }^{1}$ \\ Universidad de Valencia
}

\section{RESUMEN}

El artículo revisa los modos en que las voces y los escritos del pasado, tanto los que nos han llegado como los que podemos obtener todavía de personas vivas, pueden contribuir de manera especial al conocimiento e interpretación de la cultura escolar del pasado. Defendemos que constituyen un patrimonio histórico-educativo muy importante y valioso, que hay que salvar y aprovechar. A través del mismo se indican las potencialidades y valores de estos recursos, "egodocumentos", y también sus problemas, y por lo tanto sus limitaciones y las cautelas a tomar por el investigador. Para todo ello, el trabajo repasa y analiza diversos aspectos: el valor de los "testimonios" como objeto de interpretación; el sentido y las aportaciones de la "memoria" y de sus marcos sociales; la relación significativa que se establece entre memoria y “emoción”, una vinculación que nos permite captar y revelar "vivencias" de las prácticas escolares. El artículo acaba ofreciendo un sintético conjunto de retos y de caminos de investigación y estudio, que señalan este campo como sugerente y necesario, y que nos permitirá no solo conocimiento descriptivo sino "personalizar" a los protagonistas, contextualizarlos en su comunidad socio-política

Palabras-clave: Património-Educativo. Egodocumentos. Cultura escolar.

\section{O LEGADO DE VOZES E ESCRITOS: SEU VALOR COMO TESTEMUNHO DA CULTURA ESCOLAR.}

\section{RESUMO}

$\mathrm{O}$ artigo analisa as maneiras pelas quais as vozes e os escritos do passado, tanto os que chegaram até nós como os que ainda podemos obter de pessoas vivas, podem contribuir de maneira especial para o conhecimento e a interpretação da cultura escolar do passado. Defendemos que eles constituem um patrimonio histórico-educativo muito importante e valioso, que deve ser salvo e aproveitado. Através do mesmo são indicadas as potencialidades e os valores desses recursos, "egodocumentos", e também seus problemas, bem como limitações e cautelas a serem consideradas pelo investigador. Em todo o trabalho se repassa e analisa diversos aspectos: o valor dos "testemunhos" como objeto de interpretação; o sentido e as contribuições da "memoria" e de seus quadros sociais; a relação significativa que se establece entre memoria e "emoção", uma vinculação que nos permite captar e revelar "vivencias" de práticas escolares. O artigo termina oferecendo um conjunto sintético, de desafios e caminhos de pesquisa e estudo, que aponta esse

\footnotetext{
${ }^{1}$ Presidente da SEPHE - Sociedad Española para el Estudio del Património Histórico-Educativo.
} 
campo como sugestivo e necessário, o que nos permitirá não apenas conhecimento descritivo, mas também "personalizar" os protagonistas, contextualizando-os em sua comunidade sociopolítica.

Palavras-chave: Patrimônio-Educativo. Egodocumentos. Cultura escolar.

\title{
VOICES AND WRITINGS' LEGACY: ITS VALUE AS A SCHOOL CULTURE'S TESTIMONY
}

\begin{abstract}
The article analyzes the ways by which the voices and writings from the past, both those that have reached us as those that we still can obtain from living people, may contribute in a special way to the knowledge and interpretation of school culture from the past. We argue they make a very important and valuable historical and educational heritage, which must be saved and availed. The potentialities and values of these resources, "egodocuments", are indicated through it, and also their problems, as well as the limitations and cautions to be considerated by the researcher. Several aspects are reviewed and analyzed through the entire work: the "testimonies" value as an object of interpretation; "memory"'s meaning and contributions and its social framework; the significant relation established between memory and "emotion", a connection that allows us to capture and reveal school practices' "experiences". The article ends by offering a synthetic set, challenges and paths of research and study, that indicates this field as a suggestive and necessary one, which will enable us not only a descriptive knowledge, but also to "personalize" the protagonists, contextualizing them at their socio-political community.
\end{abstract}

Key words: Educational Heritage. Egodocuments. School culture.

\section{LE LEGS DES VOIX ET DES ECRITS: LEUR VALEUR EN TANT QUE TEMOIGNAGE DE LA CULTURE SCOLAIRE.}

\section{RÉSUMÉ}

L'article passe en revue la façon dont les voix et les écrits du passé, à la fois ceux qui nous sont parvenus et ceux que nous pouvons encore obtenir de personnes vivantes, peuvent contribuer de manière particulière à la connaissance et à l'interprétation de la culture scolaire du passé. Je tiens a dire, qu'ils constituent un patrimoine historique et éducatif très important et précieux, qui doit être sauvegardé et profité. L'écrit montre les potentialités et les valeurs de ces ressources, "egodocuments", ainsi que leurs problèmes, et donc leurs limites et les mesures à prendre par le chercheur. Pour tout cela, l'article apprendre divers aspects: la valeur des «témoignages» comme objet d'interprétation; le sens et les contributions de la «mémoire» et de ses cadres sociaux; la relation significative entre la mémoire et «l'émotion», un lien qui nous permet de capturer et de révéler des «expériences» des pratiques scolaires. L'article se termine en proposant un ensemble synthétique de défis et de pistes de recherche et d'étude, qui pointent ce domaine comme suggestif et nécessaire, qui nous amène non seulement à la connaissance descriptive mais aussi à «personnaliser» les protagonistes, les contextualiser dans leur communauté sociopolitique.

Mots-clés: Patrimoine éducatif. Egodocuments. Culture scolaire. 
La verdad es que yo guardo con cariño dentro de mí tantas cosas como me transmitieron. Creo en esa cadena que nos enlaza. Creo en la canción que se teje con las canciones que llegan de tan lejos. Creo en la memoria ancestral.

María Teresa León, Memoria de la melancolía, Losada, 1970, p.78

Claro que el problema de toda biografía radica precisamente en esto: en la conexión entre los hechos externos, objetivamente comprobables, y el sentido íntimo de la vida individual, que aun para el propio sujeto que la vive está muy lejos de ser transparente.

Francisco Ayala, Recuerdos y olvidos, Alianza, 1988, p 22

La vida no es la que uno vivió, sino la que uno recuerda y cómo la recuerda para contarla.

Gabriel García Márquez, Vivir para contarla, Mondadori, 2014, prefacio

Las huellas o muestras que conservamos de nuestro pasado educativo son preciados bienes culturales que nos transmiten un patrimonio o herencia; sin duda nos permiten comprender mejor bastantes aspectos importantes del mismo. Podemos hacerlo por medio de diversas materialidades que perduran, pero también a través de voces y escritos que vienen de aquel tiempo pretérito, o que estamos en condiciones de obtener, en la actualidad, desde la palabra viva de actores y/o testigos del mismo; en ese caso nos presentan los pasados de los vivos o las memorias de nuestros propios coetáneos. Son fuentes que transmiten personajes-narradores, que contienen materiales decisivos para el estudio de las normas y prácticas de la cultura escolar, y que nos llegan a través de historias de vida o relatos biográficos, memorias, diarios, entrevistas, registros y archivos orales, etc. Sin duda presentan problemas y limitaciones, pero también ofrecen oportunidades para abundar en esa constante búsqueda de integración entre historia, objetividad y verdad, en ese continuo procesodel que hablaba Edward Carr- entre el historiador y sus hechos.

\section{EL TESTIMONIO}

Es importante aprovechar el valor potencial de la palabra de varones y mujeres que participaron en la historia de su presente, como sujetos de hechos que fueron configurando las distintas componentes de la cultura escolar. Ellos y ellas fueron testigos y dieron testimonio en razón de su historia vivida, y de la que desearon dejar constancia; otros y otras registraron experiencias- no en el propio tiempo del acontecer, sino desde el recuerdo- y ahora pueden llegar 
a construir una concepción personal de aquellos acontecimientos, que estamos a tiempo de recoger develar y preservar. El conjunto de recursos es amplio y variado y es bueno servirse de ellos. Ya Lucien Febvre afirmó, en las primeras páginas de sus Combates por la Historia, que esta era la ciencia de los hechos humanos y que parte de ese trabajo era también "encontrar a los hombres que han vivido los hechos".

Y al fijar nuestra atención en esa voces y escritos, etnotextos, nos situamos en coincidencia con un conjunto de consideraciones actuales del trabajo historiográfico y pedagógico en busca de comprender, algo que supone asumir estos enfoques: la multiplicidad y heterogeneidad de análisis y fuentes; la indagación cualitativa; la mirada antropológica y el modelo de trabajo etnográfico; la oportunidad del paradigma de inferencias indiciales (Ginzburg, Carlo); la exigencia de narrativa e interpretación, con la presencia sólida del enfoque hermenéutico; el interés por la subjetividad y la perspectiva biográfica como recursos para hacer emerger la potencialidad significante del sujeto, en expresión de Paul Ricoeur; la revalorización de la memoria y el recurso al recuerdo y la experiencia; la orientación hacia el giro afectivo o emocional; el retorno del sujeto que tiene “experiencia vivida", y, en fin, la redefinición y recuperación de lo político.

Sobre aquel tipo de sugerentes recursos, en los que ahora nos centramos, conviene, en principio, puntualizar algunas cuestiones. Porque, ciertamente, a esas testificaciones no hay que adjudicarles, en mi opinión, valor absoluto; ponen a nuestro alcance un conocimiento fragmentario, irrelevante o cuestionable, pero con aportes y potencialidades, y por eso pretendo sostener aquí- al mismo tiempo- una llamada a contar con las importantes virtualidades que abre y a establecer las precauciones precisas para hacerlas posibles.

Es un trabajo con testimonios -narración subjetiva- al que hemos de pedir un buen número de requisitos: la dosis adecuada tanto de exactitud como de credibilidad; la utilización de procedimientos de recogida rigurosos; la mayor solicitud de objetividad; la oportuna discriminación entre las aportaciones de la memoria real, la memoria sugerida o mediatizada, la imaginada o confundida; la indagación que distinga entre el recuerdo y los procesos de codificación y recodificación que el sujeto efectúa seleccionando o interpretando; la cuidadosa detección de los elementos más frágiles o menos solventes. Importan, pues, y mucho, los usos que debemos dar al material obtenido de esos plurales testimonios, entre los que caben los provenientes de actores, de expertos, de gobernantes, etc. 
Todo lo señalado en estas últimas líneas, insistamos, apunta a valorarlas, pero también a aceptarlas con la mayor cautela y exigencia; son ciertamente unos materiales que con su relatividad, imperfección, heterogeneidad y no representatividad, demandan -como toda fuente histórica- una seria crítica.

Todavía podemos acudir a la sabia reflexión de Marc Bloch (1949) para tener en cuenta cómo esos testimonios personales resultan irremplazables para indagar las mentalidades, las sensibilidades, los que él llamó “fenómenos de consciencia”. Rigor metodológico y ético son precisos en ese intento. Digámoslo ya de entrada, lo aprendimos de Febvre y sus "Combates", entendemos la Historia como "estudio científicamente elaborado: con el sustancial trabajo de plantear problemas y formular significados, de dar luz a los hechos históricos (Febvre, Lucien, 1953)

Demos paso, concedamos confianza, prestemos atención - como escribe Beatriz Sarlo (2005, pp.21-22) a los derechos y la verdad de la subjetividad que puede guardarse en la textura de vida, en la rememoración de la experiencia. Y, en todo caso, aceptando lo anterior, pasemos a revisar, varios aspectos que - a mi juicio- es preciso respetar y conseguir realmente en el uso de estas fuentes:

a. Por una parte -como desarrollaré en otros lugares de este estudio- importa advertir que en la construcción de la experiencia, como en la configuración del conocimiento, concurren procesos, relaciones y factores sociales y políticos. Se requiere, consecuentemente, ubicar y contextualizar la dimensión personal del sujeto-testigo-narrador. Ivor Goodson lo ha escrito, hace poco, con claridad:

"En primer lugar, la historia de vida personal es un dispositivo de individualización si se divorcia del contexto. Se centra en la singularidad de la personalidad y la circunstancia individuales y al hacerlo puede obscurecer o ignorar las circunstancias colectivas y los movimientos históricos. Las historias de vida sólo se construyen en una circunstancia histórica específica y condiciones culturales -éstas tienen que obtenerse en nuestra comprensión metodológica-. En segundo lugar, la historia de vida individual, lejos de ser construida personalmente, lleva en sí misma un "guion". Los guiones sociales que la gente emplea al contar sus historias de vida se derivan de un pequeño número de arquetipos aceptables disponibles en la sociedad en general. El guion de la historia de vida, lejos de ser autónomo, depende en gran medida de los guiones sociales más amplios. En cierto sentido, lo que obtenemos cuando escuchamos una historia de vida es una combinación de historias arquetípicas derivadas de fuerzas sociales más amplias y las caracterizaciones personales invocadas por el contador de historias de vida. La historia de vida, por lo tanto, tiene que estar culturalmente 
localizada a medida que perseguimos nuestras comprensiones" (Goodsson, Ivor. 2017, p.37)

b. Desde otra perspectiva cabe anotar aquí como nueva señal esta expresión de John Beverley (1994): "El testimonio es un objeto de interpretación; no un espejo del mundo". Nos deja, así, esa tarea generadora, tener presente que ese testimonio no da una imagen completa de la realidad porque el informante no solo la describe, sino que la narra. Por consiguiente, ni hay testimonio sin experiencia ni experiencia sin narración (Sarlo, Beatriz, 2005, p.29; y que ante el testimonio debe tenerse en cuenta que, con la información o vivencia que proporciona, se hace necesario conseguir fundamentalmente una componente de explicación, que fortalezca de forma suficiente la posibilidad de objetivación y generalización. Es decir, se hace imprescindible un trabajo epistémico consistente.

Considero que Paul Ricoeur (2003) elaboró una utilísima perspectiva para actuar en tal dirección; me refiero a su manera de entender el testimonio como una estructura fundamental de transición entre la memoria y la historia. Veamos. Al situarse en la tradición aristotélica de lo persuasivo, de lo verosímil, de lo probable, busca un enlace entre el testimonio y lo que él denomina atestación, un concepto que no vincula con la certeza o la verdad, sino con una creencia, "creo en" la palabra del testigo; no proporciona exactitud, pero sí fiabilidad. Y ello le lleva a exigir el uso de herramientas críticas de contrate: qué es lo fiable, qué es lo contrastable. A esa tarea la llama "operación histórica", un camino que permita acercarse a la verificación, y que integra la explicación e interpretación que pueda convertir al testimonio en un "documento" histórico.

Todorov (2002, p.196) puede señalarnos otra indicación al respecto, en su texto Memoria del mal, tentación del bien: trata allí sobre la necesidad de examinar la especificidad de cada acontecimiento de los que obtenemos testimonio; quiere decir esto que la unicidad o singularidad de cada uno, claro, es evidente, pero lo que hay que discernir sustancialmente es "el sentido" del mismo. Un asunto, pues, esencial en aquella mencionada operación historiográfica que inexcusablemente es activa, argumentada, y crítica.

c. Sin embargo hay que añadir que - aún con esa inexcusable búsqueda de racionalidad- no puede desaprovecharse la significación que pueda representar la componente de singular subjetividad que el testigo nos revela. El carácter subjetivo y autorreferencial de estas fuentes nos sirve en tanto esa especie de analítica microhistórica deja ver pensamientos, sentimientos, vivencias, profundidades, a las que nuestro afán de comprensión no debe renunciar. Haciendo caso 
para ello a Carlo Ginzburg: experimentando a base de casos, observando meticulosamente los rastros, leyendo en los detalles.

d. Por consiguiente: ¿Cómo constituir documentos orales que puedan ser procedimientos de análisis comparables con los que surgen de las fuentes escritas? Esa es la pregunta que se hace Joutard (1986, pp. 302-311) cuando traza la ruta del investigador en este campo; solo nos detendremos aquí en un breve apunte de dos de sus observaciones: no convertir la entrevista en un interrogatorio sino hacer de ella un "verdadero diálogo", una "aventura en común"; no convertirse en un entrevistador silencioso, ni dejar al entrevistado sin objeto ni control, por lo cual hay que facilitar su iniciativa pero mantener siempre una dirección. Se trata, por lo tanto, de rescatar experiencias, reproduciendo o transcribiendo, sí, pero también de generar visiones e interpretaciones, develar la profundidad y la diversidad de dichas experiencias: sus concreciones específicas, sus detalles, la presencia de continuidades o rupturas. Es obligado que el intérprete efectúe procesos de selección, codificación y recodificación de los mensajes que vayan develando la extraordinaria complejidad de lo evocado.

e. Particular significación toma ese aspecto en el caso de la historia del presente, aquella que pierde el carácter acabado de la Historia debido a que su escritura es contemporánea con los hechos analizados. Las fuentes escritas y orales requieren sin duda un tratamiento específico que integre adecuadamente el estudio de los individuos y el del colectivo, el encuentro entre quienes producen memoria y aquellos y aquellas que producen la comprensión histórica. (Voldman, D., 2000). Y en este ámbito, también, esa egohistoria, esa "historización” de la propia experiencia de la que escribe Julio Aróstegui (2004 a, pp. 138-139) se nos ha de presentar como una excelente oportunidad de aún con todas las subjetividades- trabajar por la objetivación de la historia vivida, entrelazada con la de nuestros coetáneos, con la generación precedente y con la que está llegando ya.

f. Para ello, podemos mencionar, al menos, tres instrumentos fundamentales. Por una parte, interesa propiciar la autobiografía escrita; porque nos facilita más, como recomienda Daniel Bertaux (2005), el estimular la conciencia reflexiva del protagonista o narrador. Por otro lado, y en el caso de entrevistas individuales o realización de talleres grupales o grupos de discusión, es difícil estandarizar la fórmula de trabajo; el propio Bertaux incita a que la observación y el proceso práctico del mismo vaya convirtiéndose en creador de los modos procedimentales. Ha de ser más que interesante, en definitiva, conseguir establecer relaciones dialógicas entre protagonistas y quienes dirigen la investigación. Ellas pueden facilitarse generando una escucha empática o 
estimulando la conexión con espacios y objetos que despierten emociones y sentimientos; lo decisivo, en fin, puede ser entender y practicar esta vía metodológica desarrollando una situación y oportunidad comunicativa.

En efecto, como expresara Anthony Giddens (1990) la conversación es un proceso fundamental para producir sentido; se trata, pues, de hacer verdadera hermenéutica en la historia oral; promover encuentro y diálogo que produzca aquella "fusión de horizontes" que señala Gadamer (2002): una especie de conciliación entre el plano objetual (texto) y el subjetual (intérprete). Es el producto, en su línea, de un diálogo conversacional. Entre nosotros, Pilar García Nieto (1.988-1989) se adelantó a advertirnos de una grave cuestión: La fuente oral -escribía- no es la voz espontánea y libre de la gente que habla por sí misma; no se trata, añadía de "agarrar el magnetófono, ponerlo delante de una persona y que nos diga lo que piensa. Y concluye: "La fuente oral es el resultado de la interacción entre el historiador y las personas a las cuales entrevista, es decir, los testimonios o informantes. El historiador/a es quien va al encuentro de los testimonios, abre los temas, aporta materiales, genera estímulos. Pero al mismo tiempo es, también, quien analiza el pasado y crea la fuente a partir de un planteamiento".

Al investigador, no está de más insistir, se le exige dirección y método para implementar la adecuada hermenéutica sobre las diversas modalidades narrativas, ofreciendo explicación “convincente y no contradictoria" (Bruner, Jerome, 1991).

Pero aún nos falta añadir, como dijimos, una tercera vía, a la que aludiremos ahora con brevedad. Es un elemento motivador y tal vez con notable incidencia en la difusión pública del tema del patrimonio histórico educativo. La utilidad del testimonio ofrecido por estas fuentes orales completa de forma atractiva la presencia en los museos de lo material; es decir, serla adecuado que pensemos en una mayor musealización de dichas fuentes, poniendo el testimonio o relato de las personas en el centro de la experiencia de visita en los museos: como fuente documental, como recurso o instrumento de interpretación, como "objeto" de museo que invita a comprender sentidos, y que a -cada uno- nos deja ver una diversidad de sujetos, los otros, y de perspectivas, vivencias.( Charlebois, C. \& Leclerc, J. (2015.

Pues bien, con todo este repaso, podemos constatar cómo lo que aparece claramente en tales indicaciones es el hecho de que el testimonio no es ya el resultado definitivo; más bien se nos ofrece como un recurso -interdisciplinar, desde luego- para proseguir, se nos presenta como un valioso yacimiento a explorar y aplicar. 


\section{LA MEMORIA.}

Hay un acuerdo generalizado en que la memoria es necesaria para conocer la historia, y también se coincide en que no es suficiente. Recordando, por ejemplo, a Tzvetan Todorov (2000) coincidiremos en que su corrección va unida a que sirva a la verdad y a que el recurso a ella no caiga en usos indebidos que conduzcan a un culto de la memoria, que conllevarán un nuevo "culto" desmedido a otros tiempos y hechos, a otras cosas y personas, etc.

Pero también existe otra corriente que estima esos testimonios legados, o los que interesa conseguir desde la voz presente, todavía, de protagonistas concretos; se considera, de tal manera, que son aportaciones de la memoria_a la construcción historiográfica, aunque -conviene recordaresta se ocupe fundamentalmente por lo colectivo. Hay que valorarlos, adecuadamente, sin ninguna duda.

Y, de nuevo, es momento, sin embargo, de precisar la necesidad de cuidar atentamente algunas cuestiones al respecto:

a. Que la memoria efectúa un proceso que registra experiencias, recuerda el pasado, lo revive de alguna manera, y finalmente produce con ello una rectualización o reconstrucción del mismo. Con tal desarrollo, como apuntara Ricoeur, la memoria es también activa y reorganizadora; construye continuadamente, y da significado, "tematiza" la experiencia. Efectivamente a él le importaba cómo pasar del recuerdo a la memoria reflexiva del sujeto que recuerda. Porque no hay que olvidarlo: el testigo es ya un narrador; y, después, al historiador le corresponde "hermeneutizar" la memoria (Escolano)

\section{b. Que la memoria es diferente a la Historia}

Halbwachs, empeñado en su diálogo con Bergson y Durkheim, escribió así, en 1925, al inicio mismo de Los marcos sociales de la memoria. Sin duda su sintética expresión nos ahorra aquí más explicitaciones:

Lo más usual es que yo me acuerde de aquello que los otros me inducen a recordar, que su memoria viene en ayuda de la mía, que la mía se apoya en la de ellos (...). Puesto que los recuerdos son evocados desde afuera, y los grupos de los que formo parte me ofrecen en cada momento los medios de reconstruirlos, siempre y cuando me acerque a ellos y adopte, al menos, temporalmente sus modos de pensar (...)Es en este sentido que existiría una memoria colectiva y los marcos sociales de la memoria, y es en la medida en que nuestro pensamiento individual se reubica en estos marcos y participa en esta memoria que sería capaz de recordar(...)eso que 
llamamos los marcos colectivos de la memoria serían el resultado, la suma, la combinación de los recuerdos individuales de muchos miembros de una misma sociedad(...)...Estos marcos colectivos de la memoria no son simples formas vacías donde los recuerdos que vienen de otras partes se encajarían como en un ajuste de piezas; todo lo contrario, estos marcos son -precisamente- los instrumentos que la memoria colectiva utiliza para reconstruir una imagen del pasado acorde con cada época y en sintonía con los pensamientos dominantes de la sociedad...podemos perfectamente decir que el individuo recuerda cuando asume el punto de vista del grupo y que la memoria del grupo se manifiesta y realiza en las memorias individuales." (Halbwachs, pp. 7-11)

Esas nociones de las que nos provee la colectividad son el tiempo, el espacio, el lenguaje, los significados culturales. Disponemos así de una memoria colectiva, la de las representaciones compartidas. Una memoria que marca una distinción clara con la Historia; pues la memoria es selectiva, parcial, incompleta, y la historia es una, objetiva e imparcial, se alcanza a través de una exigente construcción racional de la memoria. Es obvio que el historiador no es un testigo, no procede como un periodista, no hace memorialismo conmemorativo o mitificador, y por lo tanto hay que reconocer que la memoria - con desinformación, olvido, distorsiones- no puede sustituir a la historia.

Y en este momento quiero evocar las palabras de Manuel Cruz (2009, p.204) advirtiendo sobre una situación a evitar en el esfuerzo por comprender el pasado, no caer en ese lugar en dónde "las certezas han sido desplazadas por las convicciones, y las pasiones o las identificaciones emotivas, incapaces de ayudarnos a interpretar el mundo (...)"

Sigamos pues, acordando posiciones respecto a esa distinción entre Memoria e Historia. Bueno será recordar a Pierre Nora, que coincidía en esa línea que constatamos, sosteniendo que «la memoria es la vida», que sufre evoluciones con el tiempo, que puede ser -incluso- inconsciente de sus propias deformaciones, que llega a ser afectada por la memoria institucional, y que se presta a diferentes tipos de manipulaciones; por el contrario, la Historia es, debe ser, «la reconstrucción siempre problemática e incompleta de lo que ya no existe».

Y si leemos a De Certeau (1984) comprobamos su idea de que es preciso producir constantemente un análisis- desde el tiempo de la información y en el empleo de los documentospara llegar a poner de manifiesto diferencias, para establecer la relación entre particularidades y regularidades, para incorporar formalizaciones científicas; todo, entiende, es producto de "una operación que se sitúa en un conjunto de prácticas", y que a través del ejercicio de métodos y 
organización obtiene un producto que significa un progreso. Las coincidencias en esa exigencia y tarea, indudablemente podrían multiplicarse; citaremos sólo tres más.

Como piensa Pomian, (2007, pp. 15-54), el concepto «historia» hace referencia a un muy diverso conjunto epistemológico de prácticas cognitivas, a una heterogeneidad que devela la historicidad misma de la historia, lograda por medio de una superposición de estratos que han ido generando y modificando significaciones. Y de nuevo, como segunda referencia, hemos de citar a Ricoeur (2003) y la “operación historiográfica” que postula en tres fases: al documento y la memoria archivada hay que unir la explicación-comprensión y la representación "escrituraria”. La clave, desde luego, es el acierto en el análisis crítico.

Por último, como tercer apunte, Julio Aróstegui (2004) escribió de forma muy acertada sobre la relación entre memoria e historia, haciéndonos ver que ante la fiabilidad que buscamos en la primera el conocimiento histórico asume como imprescindible su necesario contenido de verdad, y que por lo tanto para que la memoria sea historia necesita «algo más» que el esfuerzo por la rememoración: necesita ser convertida en «memoria anónima», en memoria objetivada. Eso ha de ser la consecución ineludible. Por lo tanto, Aróstegui, aunque reconocía la importancia que la cultura actual da a la función social de la memoria, no hacía equivalentes la búsqueda de la memoria y la lucha por la historia, y por la verdad de ésta: "Es erróneo, en fin, suponer que ambas cosas son sinónimas y que la lucha por la memoria es inequívocamente la muestra de una «persistente conciencia histórica» como «característica emblemática de nuestra condición de contemporáneos». Conservar la memoria, nos dice, no equivale- "ni indefectible ni inmediatamente", a construir historia.

La memoria, insistamos para concluir este apartado, es una fuente para la Historia, cabe una relación dialéctica entre ellas, es posible una asociación cooperativa- más que una disgregaciónque habrá que considerar incluso con sus innegables tensiones. Si para Ricoeur eran concurrentes, para Philippe Joutard (2013) la tarea consiste en articularlas.

c. Expresemos ahora que la memoria no solo es informativa, también contiene componentes relacionadas con lo emotivo; la memoria es también emoción y sentimiento. Es conocido como el neurólogo portugués, António Damásio ha abundado en la función clave que desempeña el sentimiento en las formas que van manifestando nuestro propio pensamiento, nuestras decisiones, nuestro comportamiento (Damásio, A. 1996). Para él no somos máquinas pensantes que sentimos, somos sentimientos que pensamos. Y, por otro lado, una relevante definición de las emociones 
desde la Psicología Social nos refiere que estas se dan en las interacciones individuo-mundo, como parte del proceso de objetivación; ellas crean así nuestra particular experiencia del mundo, la dotan también de sentido porque las emociones ciertamente se corresponden con objetivos, intereses, aspiraciones, apreciaciones y evaluaciones. (Tiedens, L.Z. y Leach, C.W. (2004).

En fin, aunque no es oportuno ni posible entrar aquí en todas las perspectivas desde las que se estudia el tema de las emociones (darwiniana, jamesiana, socioconstructuctivista y cognitiva), podemos indicar que todas coinciden en que la emoción es multidimensional y que con su extraordinaria complejidad proporciona respuestas en el plano cognitivo-subjetivo, en el conductual-expresivo, y en el fisiológico-adaptación. Y conviene anotar que ello convoca a considerar la intensidad y relevancia de la expresión emocional. Es, con seguridad, un objeto y recurso complejo. Y ahora mismo el avance de las neurociencias ha fortalecido esa dirección de trabajo en campos como la neuroética, la neuropolítica, también en el campo de la economía, y cómo no en el de la educación.

En este caso, particularmente, debemos, quizás, considerar como motivación estas palabras de Norbert Elias (1979): A veces- escribe- "la costumbre en la contención de las emociones llega tan lejos, que el individuo ya no tiene posibilidad de manifestar sin temer sus efectos reprimidos, de satisfacer directamente sus instintos sofocados". De esa forma, añade enseguida "aquellos impulsos concretos se acorazan de tal modo con miedos de carácter automático que... pasan toda la vida sordos y mudos (...)”. Una posibilidad sugerente más para acceder a los profundos espacios del sentido y del significado.

\section{LA EMOCIÓN.}

Anotada en principio la importancia y singularidad de ese aspecto que subraya la relación entre los fenómenos emocionales y los procesos racionales, que considera al discurso y los afectos como no excluyentes, podemos hacer a continuación un pequeño detenimiento en la "aventura" que esa implicación abre a los historiadores y a los pedagogos. No faltan bases e impulsos para ello. Desde que en 1941 Lucien Febvre publicó un artículo, en Annales, sobre "La sensibilité et l'histoire. Comment reconstituer la vie affective d'autrefois", hasta que Jan Plamper (2014) manifestara su confianza en que las emociones se conviertan en otra categoría útil de análisis histórico", o que Noah Sobe (2102) instara a buscar lo afectivo en la historia de la educación. Fijémonos, pues, en estas consideraciones para señalar lugares de indagación y reflexión:

Rev. Iberoam. Patrim. Histórico-Educativo, Campinas (SP), v. 6, p. 1-31, e020001, 2020. 
a. Constatando, en primer término, algo que viene siendo lugar común en cada uno de los epígrafes de este trabajo. Se hace obligado mencionar la existencia de numerosos estudios sobre dos temas esenciales: el papel de los contextos sociales, la cultura, el lenguaje, el poder y la ideología en la construcción de las emociones, y el papel de las mismas en la construcción de la identidad (autoestima, estilo afectivo, etc.).

Y esa incidencia sobre la construcción social de la emoción, también se atiende en la perspectiva histórica y la discursiva (Belli, Simone, 2009). Las emociones, se plantea desde esa posición, reflejan igualmente lo social, hay una dimensión profundamente social en ellas que responde a relaciones, diferencias y jerarquías, que se vincula con valores religiosos, familiares cívicos, que integra la complejidad de naturaleza y cultura, lenguaje y experiencia, individuo y colectivo. Algunos ejemplos son para nosotros, historiadores de la educación, particularmente clarificadores de esa densa trama: lo podemos apreciar cuando describimos una constante y evidente pedagogía emocional en las formas históricas de enseñanza-aprendizaje; o cuando conocemos el enorme interés del poder en producir en la institución escolar emociones, en regularlas, en el cultivo allí del sentimiento nacional o la emoción patriótica (Quentin Deluermoz, Emmanuel Fureix, Hervé Mazurel et M'hamed Oualdi ( 2013). Y teniendo en cuenta además que, en ese viaje, la historia cultural y el enfoque, tendencia o giro afectivo han impulsado un nuevo esfuerzo para la comprensión histórica de las emociones. Desde esa afirmación se colige la necesidad y utilidad de tenerlo en cuenta para profundizar en el concepto de comprensión en las Ciencias Sociales; algo que nos estimula a estudiar la dimensión afectiva que acompaña o sigue a la experiencia humana y atender a la potencialidad explicativa de los estados afectivos que interactúan en/con la capacidad cognitiva.

En el ámbito español de la Historia de la Educación el profesor Agustín Escolano nos ha ofrecido convocatoria y orientaciones, desde hace tiempo, poniendo de relieve la pertinencia de este giro afectivo en la historia de la formación humana; la cultura de la escuela, afirma, es un "conjunto holístico de cogniciones y emociones" y conviene conocer las representaciones expresas o implícitas, precisa- que quedan fijadas en la memoria de los escolares (Escolano, Agustín, 2005, 2018). Sugerente propuesta, necesaria sin duda.

b. En segundo lugar, recordando el estímulo inicial que para integrar el asunto en la historiografía significaron Febrve y Bloch, cuando incorporan en ella el interés por las mentalidades, los imaginarios, la sensibilidad, las emociones. Una dedicación sobre la que en la 
actualidad ya hay una interesante corriente historiográfica que va definiendo el ámbito de estudio (Moscoso, J., 2015; Reddy, W. M, 2001; Stearns, P.N., 2003, Stearns, P.N. y C.Z, 1985; Nicole Eustace, Eugenia Lean, Julie Livingston, Jan Plamper, William M. Reddy, Barbara H. Rosenwein, 2012). Quizás nos sea útil quedarnos, en este punto, con la esencial consideración de Reddy sobre el hecho de que la emoción contiene elementos que son como "percepciones" que no alcanzan a despertar completamente nuestra "atención", pero que producen cosas porque "activan" reacciones y afectan a nuestra relación con el entorno. Podemos llegar a observarlas como un constructo social e histórico.

c. Además, y como un tercer aspecto a reflexionar, es evidente que la tarea no es fácil, que son variados los problemas, como, por ejemplo, la propia dificultad de definir el objeto de estudio (emociones, afectos, sensibilidades); y la de afrontar la invisibilidad de los sentimientos subjetivos desde un marco analítico riguroso (Zaragoza, Juan M. 2013, Díaz Freire, José Javier, 2015). Y otro apunte más: recordando la perspectiva aristotélica, las disposiciones emocionales se nos aparecen como una tensión, una tendencia que acerca deseo y razón; el "deseo racional” y "el pensamiento deseante" de la Ética a Nicómaco. Entendamos, así, la emoción no solo como percepción sino como constituida también por un juicio o valoración., en ella concurren criterios racionales y éticos.

Está muy claro, en consecuencia, que son complicadas las cuestiones a perfilar; para resumir, me permito añadir un ejemplo más. Los estudios psicológicos resaltan en la actualidad la influencia de las emociones sobre los procesos de la memoria, pero se observa que la carga emotiva o el estado emocional del sujeto condiciona las preferencias o selecciones en el trabajo de recuperar, mantener o evocar el recuerdo. Determinados experimentos de MCM (mood-congruent memory) vienen mostrando que la asimetría del efecto de memoria congruente puede relacionarse con la influencia específica de cada estado afectivo sobre la forma en que se ha generado el procesamiento cognitivo afectando pues a la recuperación de recuerdos autobiográficos (Fernández de Castro, J,1997; Boyano, J.T., 2015).(Los estados de alegría tienden a producir valoraciones más intensas y juicios más expansivos, mientras la tristeza promueve valoraciones más ajustadas o sistemáticas).

Se ha comprobado también el hecho de que, en las entrevistas con las mismas personas, años después, se reescriben en cierto modo los recuerdos, al compartirlos con otros la crítica ha disminuido y la idealización ha ido cobrando mayor peso, transitando de la comunidad vivida a la 
ideada o a la imaginada, y de la memoria idealizada a la inducida o aprendida (Fernández-Soria, Juan Manuel, 2019). Llamativo y complejo, sin duda.

Por todo ello no está de más recordar aquí a Mercedes Vilanova (1998, 2013); la pionera en España de la historia oral, recordaba a Ronald Fraser, que buscaba siempre el "centro de gravedad" de la persona entrevistada; y la profesora catalana instaba a que al utilizar las fuentes orales eran imprescindible varias capacidades: saber escuchar y saber preguntar, oir lo que nos dicen y lo que no se nos dice por propia voluntad de interlocutor, porque no sabe expresarlo, o porque nosotros mismos no sabemos conseguirlo.

d. Finalmente, será necesario recordar a Roger Chartier (1992, pp. 81-104) en "El mundo como representación". Y es preciso advertir con claridad aquel citado enfoque construccionsta de las emociones; así sabemos que se conforman: "emotional regimes" o normas dominantes de la vida emocional (Reddy, William, 2001); “emotional communities”, formadas por grupos que se adhieren a una misma valoración y expresión de sus emociones (Rosenwein, Bárbara, 2010); y practicas o sistemas de sentimiento, “emotional practices( Scheer, Monique, 2012), que no solo producen emociones sino que emergiendo de disposiciones condicionadas por el contexto social y sus especificidades históricas y culturales que llegan a crear un cierto compromiso o adhesión práctica con el mundo. En este caso nos encontramos con algo que está en línea con el habitus de Pierre Bourdieu (1991, p.92-93), como sistema de estructuras cognitivas y motivacionales -y sus "disposiciones duraderas y transferibles"- predispuestas para funcionar como "estructuras estructurantes", es decir como principios generadores y organizadores de prácticas y representaciones.

Al finalizar este apartado de nuestra exposición parece apropiado sostener que la vinculación y articulación entre sujeto y contexto, subjetividad y mediación, memoria y emoción, cognición y volición, puede constituirse como un territorio fértil para laborar en/desde la búsqueda, de lo que Geertz (1988) calificó como “descripciones densas”; él mismo estimuló después esa especie de "historia etnografiada" (2002). Ese mundo puede ser un espacio y modo de avanzar en el campo de la multiplicidad de conceptos y componentes de estructuras de significación complejas. Enorme y diverso latifundio ese legado de escritos y voces, de ayer o de hoy, para la investigación; su extensión y complejidad, por cierto, reclaman la disolución de fronteras disciplinares. 


\section{URDIMBRES PARA LA IDENTIDAD-ALTERIDAD. LA MEMORIA DEL YO Y DEL NOSOTROS COMO OBSERVATORIO DE LA HISTORIA de la CULTURA ESCOLAR.}

Como enlace entre todo lo expresado hasta aquí y la última parte del presente estudio, tal vez esté justificado que leamos ahora estas palabras del sociólogo italiano Franco Ferraroti (1991, p.120):

“(...) no se trata de colgar mecánicamente los jirones de experiencia humana, vivida y fechada, sino más bien captar el nexo de condicionamientos recíproco que intercorre (sic) entre los diferentes niveles de experiencia y entre estos y el plano macrosistemático estructural, a fin de fijar los primeros elementos de una dialéctica relacional en la que naturaleza y cultura, ambiente e historia, sistema, clase, grupo e individuo establezcan entre sí una relación necesaria".

Desde ese recuerdo, que quiere ser señal, iniciaremos un pequeño espacio para revisitar algunos puntos que hemos ido indicando en diferentes ocasiones. A lo largo de nuestra exposición, en efecto, ha quedado bien de manifiesto el papel de "los otros" en la configuración del "yo", así como la idea de que podemos aceptar que la alteridad es un elemento o factor referencial que se integra en la constitución de nuestra propia identidad. Podríamos acordar, en resumen, que la identidad no es algo completamente cerrado y total; y que identidad y alteridad se construyen de forma relacional e histórica.

Al tomar como recurso documental la memoria de la educación no indagamos exclusivamente, por lo tanto, en una identidad aislada, sino que encontraremos perspectivas de entrecruzamientos, urdimbres, quizás imbricación. Los sujetos que nos informan respecto a su educación pueden ir confluyendo en una visión más consistente de la realidad educativa de un "nosotros" iluminador.

Recordemos otra vez a Paul Ricoeur; fijándonos concretamente, ahora, en su defensa de que la "identidad" no es solo "mismidad", la identidad es dinámica, variable, en la medida que va experimentando vivencias "a través del tiempo", va integrando exterioridades, pertenencias. Y deberemos añadir, siguiendo a Denisse Jodelet (1994) que las representaciones sociales vinculan también la experiencia personal y los modelos recibidos de la sociedad. Por otra parte, y desde el plano que desea el retorno de lo político, Chantal Mouffe (1999, pp. 138-139) entiende que la individualidad solo se construye mediante la inscripción en un conjunto de relaciones sociales. En este mismo asunto veamos aún otra forma más de expresarlo; existen "comunidades vivas de 
cultura"-en palabras de Denis Goulet-, ellas son una "racionalidad existencial", que se sostiene con tres basamentos: un sistema común de valores significativos; una base territorial, histórica, y religiosa que se comparte; y una voluntad de autoidentificación.

Y bien, citemos por último a Manuel Cruz, cuando habla de que la base de la comprensión histórica se encuentra en una socialización compartida, en la existencia de un conjunto de determinaciones estructurales fundamentales (Cruz, Manuel, 1994, p. 91). Y si los agentes a comprender- continúa- comparten con quienes se esfuerzan por comprenderlos un conjunto de determinaciones estructurales fundamentales, entonces podemos decir que la operación comprensiva resulta materialmente posible.

De forma que resulta claro: me parece que caminamos adelante desde el singular y el plural. Esta es, sin duda, una importante razón y posibilidad para trabajar sobre el conjunto de legado de voces y escritos al que aquí seguimos. En cualquier caso, además, a lo largo de nuestro recorrido hemos sido invitados muchas veces a escuchar al otro, lo que establece indefectiblemente la defensa de una relación dialógica entre testigos, testimonios e historiador.

Estudiamos esa vía reconociendo el carácter social del proceso cognitivo y del proceso individualizador; pero muy lejos de disolver el "yo". Sencillamente, entiendo que vamos a ubicarlo y distinguirlo: hemos de reconocer la particularidad y lo diferente, y el terreno o espacio de lo común. Los dos referentes se integran en nuestro interés y objetivo.

La memoria personal que se nos entrega dejará ver identidades, pero diferentes identidades, relacionadas, contratables, y compartidas a veces. Sus manifestaciones irán tejiendo y conformando una red en la que yo y nosotros se entrecruzan y/o implican. Como se ha escrito, los recuerdos pasan de ser de un conjunto de individuos a medio de construir una comunidad, de ser un conjunto de yoes a ser un nosotros (Rosa, Alberto, 2000, 2006).

Quiero apoyarme como colofón para estas coincidentes afirmaciones en la palabra certera de Emilio Lledó (1992, pp.147 y 157) cuando califica la experiencia humana como "siembra continuada", como "maduración"; dicha experiencia, agrega, está hecha por "propias determinaciones", pero nunca puede evitar "la ajena siembra de ajenas semillas". Podemos pensar, igualmente, en esto que expresa así:

«Enhebrado en el largo hilo de la escritura, ya no importa la discontinuidad de cada memoria individual que es, por su misma constitución temporal, principio y fin de su siempre limitado diálogo. En el río del pensamiento colectivo, el discurso escrito representa el cauce por donde una buena parte de ese pensamiento discurre, y por donde la finitud de cada consciencia se trasciende en la múltiple y variada 
posibilidad hermenéutica de la tradición. El pensamiento individual, hecho escritura, adquiere así resonancia colectiva. El ser concreto y personal se hace lenguaje y el diálogo, que, en cada tiempo, establece con los individuos que toman de nuevo consciencia de esa escritura, no es sino una parte de la totalidad de un inmenso dominio comunicativo que se convierte, efectivamente, en cultura (...)»

Ya en el plano más concreto de los pasos metodológicos a seguir, sólo mencionaré a Bertaux (2005) para ratificar una vez más lo que venimos refiriendo; él hace ver que las lógicas que rigen el conjunto de un mundo social se presentan igualmente en cada uno de sus componentes, y, por lo tanto, al aprovechar un relato de vida podemos pasar de la identidad particular a lo general, captamos también mecanismos sociales, procesos que reproducen y transforman; de manera que seguir una muestra de relatos nos ayuda a observar y calibrar la diversidad y diferencia de sujetos y situaciones. Y proseguir con la mirada abierta al singular y al plural. Construyendo, tal vez, interesantes materiales para una especie de memoria coral.

Así, con todo lo mencionado, podemos sostener coherentemente que las voces y escritos que nos narran el pasado pueden tener la potencialidad de develar en/para la Historia de la Educación rincones y rasgos muy profundos de la cultura empírica de la escuela. Seguramente nos faciliten la tarea de constatar las apropiaciones diferenciales o divergencias culturales a las que aludiera Roger Chartier (1992); o nos permitan descubrir esas contradicciones, esas brechas o espacios abiertos, intersticios les llamó Giovanni Levi, que existen en los sistemas normativos. ¿ Los actores o testigos no vieron otra salida más que sencillamente amoldarse, convencidos, sumisos o forzados?, ¿percibieron algún tipo de duda o presentaron alguna resistencia es los correspondientes procesos de interiorización?, ¿sintieron que la escuela ya les situaba en una experiencia cultural básica iniciática que contrastaba con aquella de la que provenían (las prácticas de lo local, la lengua propia, en su caso, las prácticas de la calle y de la familia)?, ¿constataron algún resorte que les señalara el choque con otros discursos probablemente nuevos y extraños?. En fin, busquemos, en aquellos recursos información o vivencia de fenómenos de selección, acomodación o asimilación.

Sepamos hallar en la memoria de esas voces y escritos un medio oportuno para avanzar en el análisis histórico; un trabajo que dilucide cuestiones importantes sobre los modos en que la escolaridad olvidó/rechazó/ frustró la formación de un sujeto relacional y multipolar, de unos ciudadanos insertos en la dialéctica identidad-alteridad, o posibilitados de inscribirse en el equilibrio singular-diverso-universal. Intentemos rastrear allí la presencia/ausencia de tres 
capacidades sustanciales en la arquitectura de nuestra vida, y de las que nos advierte Martha Nussbaum: las emociones, la razón práctica y la afiliación o sociabilidad. No olvidemos detectar elementos clave de legitimación de uniformidades e identificaciones impuestas y de desconsideración de otras identidades y culturas. Será decisivo, a mi modo de ver, que generemos preguntas y busquemos respuestas- sobre lo velado y lo expreso- en la experiencia que aquellas fuentes nos ofrecen.

En último término, aunque no el menos importante, considero que nos situaremos así en línea coincidente con el de un planteamiento esencial en la actualidad y del que es difícil evadirse; exactamente en la apelación que hace Pierre Rosanvallon ( 2003) a considerar y dilucidar "lo político": es decir, a reconstruir las formas con las que los individuos y los grupos han elaborado su comprensión de las situaciones, su determinación a los rechazos, las adhesiones desde las que han formulado objetivos y organizado sus acciones.

Para el avance o cumplimiento de dichos propósitos algunos ámbitos de estudio tienen un sentido relevante. Anotemos, como muestra, un pequeño grupo de ellos.

a. Precisar la función que las ideas pedagógicas han atribuido a los procesos de individuación, autodeterminación y autorrealización. Son con toda seguridad aspectos esenciales, teniendo en cuenta que producen la construcción de la identidad personal; y al mismo tiempo son los que han permitido o impedido resolver las plurales dimensiones del yo a través de una identidad dinámica, interactiva, dialógica, divergente.

b. Es recomendable, igualmente, observar con precisión las formas en las que el sistema educativo ha ido dirigido a ser muestra, prueba y legitimación de un ethos cultivado, distinto y distante. Puede ser apasionante, aunque complicado, explorar y hacer visible en el ejercicio de la rememoración las huellas que, por conducto del proceso educativo, ha dejado históricamente una especie de sociocentrismo radicado en la acción de una hegemónica identidad de clase; esa pretendida identidad modélica postuló y legitimó su superioridad y verdad, representando acontecimientos y conceptos únicamente desde una solo perspectiva; y todo en busca de una aparente homogeneidad cultural. Se trata de dimensiones, indudablemente, de un currículum oculto, pero ¿Convencían, o vencían? ¿Acaso algunos escolares pudieron, en ese mismo tiempo o después, concienciar la fractura entre enseñanza y experiencia?

c. Interesa conocer las formas y consecuencias de aquello que Thomas Popkewitz (2003) denomina como prácticas o sistemas de gobernación y disciplina; aquellas intervenciones que van 
relacionando de esa forma las racionalidades políticas con la conciencia individual, y generando, no sólo conocimiento, sino "conciencias y sensibilidades acerca del mundo". Habrá que atender, por ello, a ciertas circunstancias en las que por medio de la institucionalización educativa - con sus prácticas culturales y mecanismos reguladores- se ha instrumentalizado la identidad en una determinada dirección: bien como razón o pretexto que degeneraba en estricto control de la posible disidencia o transgresión; bien como elemento clave de legitimación socio-política; o incluso como injustificado particularismo que olvidaba otras complejas articulaciones, generando exclusión desde un pretendido y/o aparente consenso en una sociedad que, pese a todo, era diferenciada y con graves fracturas y conflictos sociales.

d. También es importante analizar los modos y efectos producidos por las "otras" educaciones, explícitas o implícitas, pero con una intencionalidad básica: las ocasiones de sociabilidad en el centro escolar, la formación del carácter, el tratamiento de la educación emocional, las enseñanzas y vivencias socializadoras del civismo o la "urbanidad", la formación moral y religiosa, los lugares y dominios en los que se situó -teórica o ejemplarmente- el sentimiento, la libertad, la conciencia, la dignidad, los derechos, la igualdad, la simpatía como comunidad de sentimientos, o el encuentro. En fin, busquemos los signos de cómo actuaban los proyectos educativos para entender al otro, que es también un yo. ¿Pasaron los cursos sin llegar a esas imprescindibles "lecciones"? o, ¿acaso algunas personas consiguieron sentir, al menos, esas llamadas por la acción de un "maestro" o "maestra"? ¿Se reiteraron, en cambio, otras máximas morales, "consignas" o preceptos, tales como la autoridad, la jerarquía y la distinción, la obediencia, el conformismo y la resignación, el orden, los deberes? ¿Qué incidencia tuvo la instrucción religiosa en la vivencia sentida y la "encarnación” práctica de la fe? ¿Cuáles fueron efectivamente los resultados del "uso interesado" de las lecciones de Geografía humana, de la Literatura, o de la componente económica? (Hernández Laina, 2018).

Nos encontramos, con todo ello, ante diferentes perspectivas que confrontan identidad y alteridad o que hacen ver su relación dialéctica, y que requieren todavía resolver incógnitas que perduran; entre ellas está el obtener testimonios de los registros metacognitivos necesarios para dar luz sobre el influjo de emociones y sentimientos en la construcción de la sociabilidad educativa y en las redes sociales de convivencia (Escolano, 2018, pp.55-56.)

e. Es necesario entrar en el tema de las narrativas nacionales, sobre todo en tiempos que impulsaban una visión esencialista de la mismas y de utilización de elementos emocionales para 
producir identidad nacional. Habrá que explorar las razones y emociones que actuaron desde la escuela en la interiorización de ideas y obligaciones referidas a la nación, a la madre patria. Se trata de una línea investigadora que debe activar diferentes miradas: para revelar visiones miopes, simplificadas y distorsionadas en la enseñanza de la Historia, una disciplina sostenida como "Biblia secularizada", en expresión de José María Jover o en "historia como terapia", en el decir de Carolyn Boyd; para detectar ausencias y presencias, excesos e insuficiencias de la socialización política, o incluso descubrir los efectos de la relevancia otorgada en determinados periodos a la dimensión afectiva de dicha socialización (Mahamud, Kira, 2012); con el objeto de precisar usos e impactos de la exaltación emocional y simbólica de la nación y el nacionalismo, la raza, la continuidad tradicional, la España genuina, o la diferente, con marcados antagonismos y severas exclusiones de otros más, los elementos extranjerizantes que intentan disolvernos, la hostilidad internacional que amenaza... o al contrario la inferioridad de otros que nos justifica para "protegerles"; para observar la minusvaloración y ocultamiento que se hace de ciertos componentes en la narración de esa "comunidad imaginada" (Anderson, Benedict, 1993) o "nación inventada"( Gellner, Ernest, 1988).

¿Podremos de tal forma constatar mejor los éxitos y fracasos de esa intencionalidad instructiva/formativa, apreciados en términos de apropiación, adhesión sentida o mayor persistencia? Es decir, ¿podremos constatar la escasa efectividad de dichos intentos de socializar la identidad nacional? ¿Los héroes y santos llegaron a ser modelos de una exaltada simbiosis religión-patria, o se convirtieron como casi todo en una más de las obligadas ritualizaciones o memorizaciones mecánicas? ¿Los mitos dejaron huellas, o quedaron reducidos a abstractos rituales, o quizás simplemente fijados en la superficialidad de unas letras extrañas? Y una pregunta más, de la que ya vamos obteniendo interesantes respuestas: ¿Qué marco interpretativo y de representación simbólica fueron capaces de conformar los alumnos por medio de las enseñanzas históricas? (Souto González, Martínez Valcárcel, Parra Monserrat, 2014).

El profesor David Parra, y como fruto de una investigación seria y de gran utilidad historiográfica y pedagógica lo ha subrayado de forma clara, mostrándonos una virtualidad más del análisis de tales recuerdos. Solo explicitando -escribe- el proceso de construcción de estos imaginarios sociales y contribuyendo a una lectura crítica de los mismos y de sus usos públicos conseguiremos configurar nuevas representaciones que posibiliten definir e implementar una educación histórica alternativa. (Parra, David, 2018, p.73) 
f. Y no podemos olvidar el objetivo de introducir aquí el género como categoría analítica de la Historia. Se tratará, en este caso, de indagar las evidencias que muestren la histórica construcción psico-social y cultural de lo femenino y de lo masculino; y por consiguiente la representación o pensamiento social y la socialización diferencial que los docentes, los libros, los escenarios y las prácticas han ido creando a través del proceso histórico de la escolarización: con la transmisión de imágenes tradicionales de la mujer, sus plurales formas de subordinación, y el establecimiento del varón como paradigma central. Buscaremos en el legado de la memoria las numerosas formas en que el tiempo escolar incidió en el conjunto de creencias, sentimientos, valores, actitudes, conductas y actividades que diferencian a hombres y mujeres; conoceremos las maneras con las que esa enseñanza les asignó determinados y distintos comportamientos, que fortalecían, a su vez, la jerarquía y la desigualdad. ¿Servirá esa memoria para conocer con mejor fundamento los efectos reales que ello tuvo en la configuración de subjetividades y en las formas de concebir al otro y al conjunto social?

g. Sobre todo en lo que concierne a la historia de tiempo presente hay dos perspectivas que debemos contemplar, cerrando así la presente muestra de tareas. En cuanto a la primera, será sugerente y eficaz estudiar las aportaciones que pueden conseguirse desde el estudio de la identidad profesional de los educadores que reconstruyen trayectorias y posiciones biográficoexperienciales; ellas, como señala Goodson (2014, p. 44), nos dejarán ver al individuo en relación con la historia de su tiempo, y por lo tanto trazarán una intersección de la historia vivida con la historia social, "cartografía social del sujeto" y "genealogía del contexto" (2012).

Hay que desarrollar este extraordinario recurso para que nos ofrezca conocimiento de esa dimensión personal ubicada o contextualizada en sus vinculaciones socio-políticas. En ese campo conviene resaltar el trabajo realizado entre nosotros por el profesor Antonio Bolívar. Desde un seguimiento amplio de la literatura sobre el tema nos ha ofrecido una clarificación estimulante para considerar la genealogía de una vida profesional, en relación con la del propio sistema educativo. Claro que, Bolívar subraya en todos sus trabajos, con acierto y fuerza, la necesidad de conjugar lo personal y lo político en esa investigación, un objetivo que compromete a considerar: la pertenencia o referencia a otros grupos de los contextos sociales, el conjunto de saberes que fundamentan la práctica, las condiciones en las que se ha ejercido, el estatuto profesional o social alcanzado en ese tiempo por la función docente. Todo ha de ser cuidadosamente enlazado para que pueda contribuir a enfocar mejor el decisivo asunto de las transformaciones a realizar en las concepciones y estilos docentes. 
Y bien, la segunda de las perspectivas a las que aludíamos, también para la historia del tiempo presente y en el campo de la educación, consiste en abrirse a otros agentes y territorios de las decisiones y las prácticas en el sistema educativo: políticos de la educación, agentes sindicales, administradores, directores y supervisores, expertos en Pedagogía, profesionales que han trabajado en ICES, animadores y gestores de las diferentes instancias de participación social en la educación, orientadores escolares, directivos y asesores de centros de formación permanente del profesorado, miembros de movimientos de renovación pedagógica. Parece claro que nos encontraremos así con otras razones, vivencias, testimonios en primera persona de estructuras, procesos y respuestas; en muchos territorios de profesionalidad pedagógica: modernización metodológica, racionalidad tecnológica, apoyo psico-pedagógico, pedagogía inclusiva, problemáticas de las reformas educativa...deseos, impulsos, limitaciones, contradicciones, resistencias insuficiencias, frustraciones, resistencia y logros.

Creo que queda claro, diversas memorias e historias, todavía no narradas, están esperando a manifestarse. Todo un testimonio repleto de razones, emociones, vivencias. Un magnífico y sugerente itinerario puede discurrir, en el caso de España, entre 1970 y 1990, y en el que voces y escritos han de contribuir a historiar algunos temas esenciales; tres, sobre todo, a mi juicio: el desarrollo de políticas democratizadoras, la racionalización y modernización educativa, la construcción de una nueva cultura pedagógica y profesional. En otros países iberoamericanos, también esos tiempos y tareas históricas aguardan una mejor clarificación.

\section{ENTRE LA AFIRMACION Y EL RETO...}

En paralelo con el reconocimiento expreso de que los testimonios son cambiantes, contradictorios, fragmentarios, notemos, debidamente también y una vez más, su potencialidad informativa, y su capacidad de transmitir vivencias particulares y significativas; algunos rincones que la vía estrictamente científica difícilmente resolverá. Incluso no estará de más servirnos aquí de otra apreciación de Todorov (2000, pp. 31 y 43); describe la "memoria literal", que convierte el recuerdo de los acontecimientos en algo singular, no comparable, pero nos expone también el sentido de la denomina "memoria ejemplar", a la que atribuye la capacidad de servir de modelo para comprender situaciones nuevas - con agentes diferentes- y, en consecuencia, aprender lecciones del pasado para actuar en el presente. Me parece que ese efecto puede ayudar a iluminar 
temas importantes de la historia de las ideas y las actividades educativas, y desde esa mejor comprensión de su evolución, procesos, continuidades y cambios, clarificar también otras cuestiones teóricas y prácticas de la educación y la Pedagogía.

A mi juicio los testimonios y la memoria manifiestan implicaciones de la experiencia individual con dimensiones que pertenecen claramente al contexto y la identidad colectiva. Extender su muestra y profundizar en ellos nos facilitará conocer, pues, la multiplicidad de los sujetos. Nos develan singularidades y similitudes, en el terreno de lo educativo, y por tanto sus efectos sobre la materialización de la diversidad y la articulación en el tejido social.

Si consideramos que con respecto a la identidad y la alteridad hay una amplia coincidencia en que ambas se construyen y reconstruyen, y que consecuentemente se configuran de una manera relacional e histórica, parece aceptable confiar en la posibilidad de que los testimonios y memorias sean útiles para precisarlas por medio de lo que nos descubren las operaciones discursivas que el narrador y el intérprete realizan en su labor investigadora.

En este sentido me parece adecuado valorar y tratar ajustadamente el aporte de la perspectiva biográfica y la historización de las experiencias individuales, la sugerente visión de las vidas "vividas" en las que "lo identitario" resulta ser un modo de coexistencia de lo singular y lo compartido.

Acabemos reafirmando que testimonio, memoria y emoción ofrecen, a quienes trabajamos en la historia, la memoria y el patrimonio educativo, un reto de conjunción, transversalidad e integración. Contamos así con un camino abierto, tal vez lleno de imprevistos, y podemos encontrar en él un espacio poliédrico y complejo, desde el que -como deseara Hanna Arendt- colaboremos en construir historia cooperante a des-individualizar lo personal y personalizar lo socio-político. No están de más las tentativas.

Confiemos en que ese reto de optimizar tales recursos permitirá progresar en dos direcciones. La primera: que desde esa conexión con "lo personal" contribuyamos a cumplir unas básicas orientaciones para la Historia, hacer saber, hacer comprender, y hacer sentir (Pomian, Krzystof,2007); en todo caso, que ese trabajo específico sobre parte de nuestro patrimonio histórico-educativo incida igualmente en el siempre pretendido objetivo de aprender a pensar históricamente y constituir conciencia histórica: evaluar críticamente estructuras y contextos, analizar cambios y continuidades, razonar causalmente. La segunda: que obtengamos resultados; no definitivos, sin duda, pero resultados en forma de útiles efectos generadores; porque a mi modo 
de ver en nuestro campo de lo educativo, y más aún desde la presencia de las personas, el avance en el conocimiento no está hecho solo con irrefutables y definitivas verdades.

Parafraseando a Lledó, hagamos que fructifiquen las voces y escritos de la memoria, y las experiencias vividas que transmiten; avancemos por ese camino, sabiendo que ese efecto no es solo "realizar el inalterable fruto", sino igualmente conseguir funcionar como "estímulo de inesperadas y originales cosechas", (1992, p.179).

\section{REFERENCIAS BIBLIOGRÁFICAS}

ANDERSON, Benedict (1993). Comunidades imaginadas. Reflexiones sobre el origen y difusión del nacionalismo. México: Fondo de Cultura Económica.

ARENDT, Hanna (1993). La condición humana. Barcelona, Paidós.

ARÓSTEGUI, Julio (2004 a). La Historia Vivida. Sobre la Historia del Presente. Madrid: Alianza Editorial.

ARÓSTEGUI, Julio (2004 b). "Retos de la memoria y trabajos de la Historia", en Pasado y Memoria. Revista de Historia Contemporánea, n³, pp. 15-36.

BELLI, Simone (2009). "La construcción de una emoción y su relación con el lenguaje:revisión y discusión de un área importante de las ciencias sociales”, en Theoria, vol. 18., n 2, pp.15-42.

BERTAUX, Daniel (2005). Los relatos de vida. Perspectiva etnosociológica. Barcelona: Ediciones Bellaterra.

BLOCH, M. (1949). Apologie pour l'histoire ou le mètier d'historien. Paris: A. Colin.

BOYD, Carolyn P. (2000). Historia Patria. Política, historia e identidad nacional en España:1855-1975. Barcelona: Pomares-Corredor.

BOLÍVAR, Antonio (2012). Metodología de la investigación biográfico-narrativa: Recogida y análisis de datos.En Passeggi, M.C. y Abrahao, (Org.).Dimensões epistemológicas e metodológicas da investigação (auto)biográfica. Porto Alegre: Editoria da PUCRS, t. II, p. 79109.

BOLÍVAR, Antonio (2014). Las historias de vida del profesorado: voces y contextos. Revista Mexicana de Investigación Educativa, núm. 62, p. 711-734.

BOLÍVAR, Antonio, Domingo, Jesús y Fernández, Manuel (2001): La investigación biográficonarrativa en educación. Madrid: La Muralla, 2001. 
BOQUET, Damien, Nagy, Piroska (2011). "Une histoire des émotions inacarnées". En Medievales, 61, pp.5-24.

BOURDIEU, Pierre (1991). El sentido práctico. Madrid: Taurus.

BOYANO, José t. y Mora, Juan A. (2015). “Estado de ánimo y memoria autobiográfica: efectos sobre la intensidad de los recuerdos escolares”. En Anales de Psicología, vol.31, n 3.

BRUNER, Jerome (1991). Actos de significado. Más allá de la revolución cognitiva. Madrid: Alianza.

CHARLEBOIS, C. \& Leclerc, J. (2015). “Les sources orales au coeur de l'exposition muséale. L'expérience du Centre d'histoire de Montréal". En Revue d'histoire de l'Amérique française, $\mathrm{n}^{\circ}$ 69 pp. 99-136.

CHARTIER, Roger (1992). El mundo como representación. Estudios sobre historia cultural. Barcelona,: Gedisa.

CRUZ, Manuel (2009). "El pasado en tanto que territorio de un conflicto". En Daímon. Revista Internacional de Filosofía, no 47, pp.193-206).

CRUZ, Manuel (1994). “Comprensión histórica, acción e identidad”. En Anales del Seminario de Metafísica,n ${ }^{\circ} 28$, pp.85-101.

DAMÁSIO, António (1996). El error de Descartes, la emoción, la razón. Barcelona: GrijalboMondadori.

DE CERTAU, Michel (1984), "La operación histórica”. En Le Goff, Jacques, y Nora, Pierre.Hacer la Historia. Nuevos problemas. Barcelona: Laia.

DELUERMOZ, Quentin, Fureix, Emmanuel, Mazurel, Hervé et M'hamed Oualdi (2013), «Écrire l'histoire des émotions : de l'objet à la catégorie d'analyse », Revue d'histoire du XIXe siècle [En ligne], 47 | 2013, mis en ligne le 31 décembre 2016, consulté le 20 mai 2019. URL: http://journals.openedition.org/rh19/4573, DOI : 10.4000/rh19.4573

DÍAZ SÁNCHEZ, Pilar y Gago González, José Maria (2006). La construcción y utilización de las fuentes orales para el estudio de la represión franquista. En Hispania Nova. Revista de Historia Contemporánea, $n^{\circ} 6$, http://hispanianova.rediris.es

DÍAZ FREIRE, José Javier (2015). “Presentación” el dossier “Emociones e Histroia". En Ayer, no 98 , pp.13-20.

ESCOLANO, Agustín (2005). "Memoria, identidad y diferencia en la construcción del campo intelectual de la educación y de su historia". En Revista de Ciencias de la Educación, n 202, pp.219-240. 
ESCOLANO, Agustín (2017). La escuela como cultura. Experiencia, memoria, arqueología. Campinas (Brasil): Alínea ed., p.325.

ESCOLANO, Agustín (2018). "El giro afectivo en la historia de la formación humana. Memoria de la escuela y emociones". En Historia y Memoria de la Educación, n 7, pp.391-422.

Escolano, Agustín (2018). Emociones \& Educación. La construcción histórica de la educación emocional. Visión Libros- CEINCE.

EUSTACE, Nicole; LEAN, Eugenia, LIVINGSTON, Julie; PLAMPER, Jam;

REDDY, William M.; ROSENWEIN, Barbara, H. (2012). AHR Conversation: The Historical Study of Emotions. En The American Historical Review, Vol. 117, n 5, pp. 1487-1531.

Disponible em: https://doi.org/10.1093/ahr/117.5.1487

FERNÁNDEZ-SORIA, Juan Manuel (2019). “Un enfoque socio-pedagógico de excepción.El Instituto para Obreros de Valencia, comunidad ideada, comunidad vivida". En Mayordomo Pérez, Alejandro y Paya Rico, Andrés, Pedagogia. Pensament, política i práctica. Lectures històriques en l societat valencia contemporània. Valencia. Tirant Humanidades, pp.27-58.

FEBVRE, Lucien (1941) «Comment reconstituer la vie affective d'autrefois? La sensibilité et l'histoire ", Annales d'histoire sociale, III, 1941; también, Combats pour l'histoire, Paris, Armand Colin, 1992, p. 221-238.

FERNÁNDEZ-CASTRO, J.; GRANERO, R.; BARRANTES, N.; CAPDEVILA, A. (1997). Estados de ánimo y sesgos en el recuerdo: papel del afecto. Psicothema, nº 9, 247-258.

GADAMER, Hans Georg (2002). Verdad y método. Salamanca: Sígueme.

GARCÍA-NIETO PARÍS, Ma del Carmen (1988-1989). “Fuentes orales e Historia”. En Studia historica. Historia contemporánea, nº 6-7, p. 105-111.

GEERTZ, Clifford (1988). La interpretación de las culturas. Barcelona: Gedisa.

GEERTTZ, Clifford (2002). Reflexiones antropológicas sobre textos filosófico. Barcelona: Paidós.

GELLNER, Ernest (1988). Naciones y nacionalismos. Madrid: Alianza editorial

GERGEN, K.J. (1985). The social constructionist movement in modern psychology. American Psychologist, no 40, 266-275.

GIDDENS, Anthony y Turner, Jonathan. (edts,). (1990), La teoría social hoy. Madrid: Alianza.

GINZBURG, Carlo (1989). Mitos, emblemas e indicios: morfología e historia. Barcelona: Gedisa. 
GOODSON, Ivor (2003). Professional Knowledge, Professional Lives Stdies of Education and Teaching, Open University Press:Buckingham.

GOODSON, I. (2003). "Hacia un desarrollo de las historias personales y profesionales de los docentes”. Revista Mexicana de Investigación Educativa 19(2003) 733-758.

GOODSON, Ivor (2004). Historias de vida del profesorado. Barcelona: Octaedro.

GOODSON, Ivor. (2017). "El ascenso de la narrativa de vida”. Revista de Investigación Cualitativa 1, 2017, pp.27-42.

HALBWACHS, Maurice (2004). Los marcos sociales de la memoria. Barcelona: Anthropos Editorial.

HERNÁNDEZ LAINA, Yovana (2018). Cultura económica y socialización política en los manuales escolares del tardofranquismo y la Transición española (1960-1985). Tesis doctoral, Universidad Nacional de Educación a Distancia.

IBÁÑEZ, T. (1988). Ideologías de la vida cotidiana. Psicología de las representaciones sociales. Barcelona: Sendai.

JODELET, Denis. (1986). La representación social: fenómenos, concepto y teoría.

En: MOSCOVICI, S. (comp.). Psicología social II. Pensamiento y vida social. Psicología social y problemas sociales. Barcelona: Paidós.

JOUTARD, Philipe (1986). Esas voces que nos vienen del pasado. México:1986.

JOUTARD, Philipe. (2013). Histoire et mémoires, conflits et Alliance. Paris: La Découverte.

MOSCOSO, J. (2015). “La historia de las emociones ¿de qué es historia?”, en Vínculos de Historia, $\mathrm{n}^{\mathrm{o}} 4$, pp.15-27.

LEVI, Giovanni (1993.) "Sobre microhistoria”, en Burke, P. , Formas de hacer Historia. Madrid:Alianza.

LLEDÓ, Emilio (1969). “Tiempo e Historia". En Boletínxdel Instituto de Estudios Helénicos, $\mathrm{n}^{\circ}$ 2, pp. 23-38.

LLEDÓ, Emilio(1992). El surco del tiempo; meditaciones sobre el mito platónico de la es critura y la memoria. Barcelona:Crítica.

MAHAMUD ANGULO, Kira (2012).Adoctrinamiento emocional y socialización política en el primer Franquismo (1939-1959): emociones y sentimientos en los manuales escolares de enseñanza primaria. Tesis doctoral, Universidad Nacional de Educación a Distancia 
MAYORDOMO, Alejandro (1995). Socialización, educación social y clases populares. Universidad de Valencia.

MAYORDOMO, Alejandro. (2004). "La construcción histórica de la identidad y la diversidad". En Bordón. Revista de Pedagogía, vol.56, n¹, pp.49-64.

MAYORDOMO, Alejandro, y Fernández Soria, Juan Manuel (2008). Patriotas y ciudadanos. El aprendizaje cívico y el proyecto de España. Valencia: Tirant lo Blanch.

MOUFFE, Chantal (1999). El retorno de lo político. Barcelona: Paidós.

NORA, Pierre (1988). "Memoria colectiva”, en LE GOFF, J; CHARTIER, R. y REVEL, J. La Nueva Historia. Bilbao: Ed. Mensajero.

NUSSBAUM, Martha (2012). Crear capacidades: propuesta para el desarrollo humano . Barcelona, Paidós.

PARRA MONSERRAT, David (2018). "El peso de la tradición en la representación y los usos públicos de la Historia escolar”. En Delgado, Ander, y Rivera, Antonio, ¿Qué saben nuestros jóvenes? Enseñanza de la Historia e identidad nacional. Granada: Comares, pp.55-75.

PLAMPAR, Jan (2014). "Historia de las emociones: caminos y retos". En Cuadernos de Historia Contemporánea, vol. 36, pp. 17-29.

POMIAN, Krzystof (2007). Sur l'histoire. Madrid: Cátedra.

POPKEWITZ, Thomas, S. (2003). "La producción de razón y poder:historia del curriculum y tradiciones intelectuales”. En Popkewitz, y otros, Historia cultural y educación. Ensayos críticos sobre conocimiento y escolarización. Barcelona: Ediciones Pomares. pp. 146-184.

REDDY, W. M., (2001): The Navigation of Feeling. A Framework for the History of Emotions, New York, Cambridge University Press.

RICOEUR, P. (2003). La Memoria, la Historia, el Olvido. Madrid: Trotta.

RICOEUR, Paul. Historia y narratividad. Barcelona: Paidós, 1999.

RICOEUR, Paul. Tiempo y narración I (configuración del tiempo en el relato histórico); II (configuración del tiempo en el relato de ficción); III (el tiempo narrado). México: Siglo XXI, 1995-96 [ ed. port.: Tempo e narrativa, 3 vols. São Paulo (Campinas: Papirus, 1994-9].

RICOEUR, Paul. Sí mismo como otro. Madrid: Siglo XXI, 1996 [trad. port.: O si-mesmo como um outro, Campinas: Papirus, 1991].

RICOEUR, Paul. La vida: un relato en busca de narrador. Ágora. Papeles de filosofía, v. 25, n. 2, p. 9-22, 2006. 
ROSA, A, y otros (2000). Memoria colectiva e identidad nacional. Madrid: Biblioteca Nueva.

ROSA, A. (2006). "Recordar, describir y explicar el pasado ¿qué, como, para el futuro de quién?", en Carretero, M. y otros (coord.). Enseñanza de la historia y memoria colectiva. Buenos Aires: Paidós, pp.41-51.

ROSANVALlON, Pierre (2003). Por una historia conceptual de lo político. México: Fondo de Cultura Económica.

ROSENWEIN, Barbara H. (2010). "Problemems and Methods in the History of Emotions" en Passions in Context I, $n^{o}$ 1, pp. 1-33.

SARLO, Beatriz (2005). Tiempo pasado. Cultura de la memoria y giro subjetivo.Una discusión. Buenos Aires: Siglo XXI editores Argentina.

SCHEER, Monique (2012). "Are emotions a kind of practice (and is that what makes them have a history)? A Bourdieuian aproche to understanding emotion", History and Theory, 52, 2, pp.193220.

SOBE, Noah. W. (2012). "Researching emotion and affect in the history of education'.En History of Education, vol 41, pp.689-695.

SOUTO GONZÁELES, Xose Manuel, Martínez Valcárcel, Nicolás, Parra Monserrat, David (2014). "La representación poliédrica de la memoria escolar: un proyecto de investigación sobre las clases de Historia de España. En Martínez Valcárcel, N. La historia de España en los recuerdos escolares: análisis, interpretación y poder de cambio de los testimonios de profesores y alumnos. Valencia: Nau Llibres, págs. 29-52.

STEARNS, Peter. N. (2003): "The Navigation of Feeling: A Framework for the History of emotions", Journal of Interdisciplinary History, 33, 3, 473-475.

STEARNS, Peter. N. y Stearns, Carol. Z., (1985): "Emotionology: Clarifying the History of Emotions and Emotional Standards', American Historical Review, 90, 4, 813-836.

THIEDENS, L.Z. y Leachh, C.W. (2004). The Social Life of Emotions. Cambridge University Press.

TODOROV,Tzvetan (2000). Los abusos de la memoria. Barcelona:Paidós Ibérica.

TODOROV, Tvetan (2002). Memoria del mal, tentación del bien. Indagación sobre el siglo XX. Barcelona: Península.

VELASCO, H. y Díaz, Á. (2007). La lógica de la investigación etnográfica. Un modelo de trabajo para etnógrafos de la escuela, Madrid: Editorial Trotta. 
VILANOVA RIBAS, Mercedes. (1998). "Reflexiones en torno a las fuentes orales y a la Historia sin adjetivos". En Estudis. Revista de Historia Moderna, 24, pp.495-504.

VILANOVA RIBAS, Mercedes (2016). "Las fuentes orales”. En Historia, Antropología y fuentes orales, $\mathrm{n}^{\circ} 49$, pp.11-14.

VOLDMAN, DANIELLE (2000). "Le témoignage dans l'histoire de présent", Bulletin de l'Institut d'histoire du présent, $\mathrm{n}^{\circ}$ 75,p. 4154. También interesa de la misma autora : "La bouche de la verité? La recherche historique et les sources orales, Les cahiers de l'IHTP, ${ }^{\circ} 31$.

ZARAGOZA BERNAL, Juan Manuel (2013). "Historia de las emociones: una corriente historiográfica en expansión", en Asclepio. Revista de Historia de la Medicina y de la Ciencia, 65, no 1: e012, doi:http//dx.doi.org/10.389. Asclepio.2013.12

Recebido em: 22 de abril de 2020

Aceito em: 30 de abril de 2020 